\title{
Posttraumatic stress disorder: possibilities for olfaction and virtual reality exposure therapy
}

\author{
Mary P. Aiken • Mike J. Berry
}

Received: 23 February 2014/ Accepted: 1 February 2015/Published online: 11 February 2015

(C) The Author(s) 2015. This article is published with open access at Springerlink.com

\begin{abstract}
Visual and auditory information has dominated the field of virtual reality (VR). Evaluation of the role of sensory stimulation in VR has highlighted olfactory stimulation as a potentially powerful yet underutilized therapeutic tool. Early studies of immersive environments, which were run as experiments, incorporated smell in the virtual experience; however, olfaction in virtual environment design and development has arguably failed to maintain a position commensurate with its sensory capacity, exemplified by the paucity of research and possible application. A review of the literature suggests that olfaction as a component of virtual environment exposure therapy may be a useful addition in the treatment of posttraumatic stress disorder (PTSD) a mental health condition triggered by a terrifying event, either experiencing or
\end{abstract}

This paper is based on research first undertaken at IADT.

\section{P. Aiken $(\square) \cdot$ M. J. Berry}

CyberPsychology Research Centre, Institute of Leadership at the Royal College of Surgeons in Ireland (RCSI), Reservoir House, Ballymoss Road, Sandyford, Dublin 18, Ireland

e-mail: maryaiken@rcsi.ie

\section{P. Aiken}

Dr. Steve Chan Center for Sensemaking, Asia-Pacific Institute for Resilience and Sustainability (AIRS), Hawaii Pacific

University, Honolulu, HI, USA

\section{P. Aiken}

Swansea University, Swansea, UK

\section{P. Aiken}

European Cyber Crime Centre (EC3), Europol, The Hague,

The Netherlands

\section{P. Aiken}

Middlesex University School of Law, London, UK witnessing it. Symptoms may include flashbacks, nightmares and anxiety, as well as uncontrollable thoughts about the event. However, to investigate the role of olfaction further research is required in the formulation, display, staging and customization of scent, coupled with an indepth analysis of the role of olfaction in cognitive function, memory, emotion and creation of presence, particularly in the context of VR treatment of PTSD. Benefits of olfactory therapy may, however, be compromised by the fact that olfactory identification deficit has been noted as a component of PTSD. Investigation is required into causative or reactive mechanisms that may underlie olfactory deficits and into suitable VR therapeutic protocols that could be designed to address these deficits. Additionally, ongoing VR technological developments may deliver increasing affordability and portability in terms of VR treatment options, particularly regarding head-mounted display units. A cyberpsychological consideration of the problem of PTSD, that is, an inter-disciplinary approach combining technology and psychology learning's may merit consideration. A review of findings suggests that research protocols focused on olfaction as a variable in a multi-sensory VR exposure therapeutic program may positively impact on treatment outcomes in PTSD population.

Keywords Virtual reality exposure therapy . Posttraumatic stress disorder - Olfaction · Odor · Memory

\section{Introduction}

"And we forget because we must, and not because we will"

Matthew Arnold, Absence (st. 3, 1852). 
While visual and auditory information has dominated the field of virtual reality (VR) to date, it can be argued that olfaction may have a vital role to play in virtual reality therapy. According to Chen (2006), "scents are extremely evocative in the virtual world, they can shift attention, add novelty, enhance mental state and add presence" (p. 580). Additionally, odor can facilitate recall (Larsson 1997), thereby having potential to address longstanding memory retrieval issues concerning traditional exposure therapy.

Posttraumatic stress disorder sensory graded immersion; a step-by-step staging process in VR has been explored by a number of researchers. The Josman et al. (2008) simulation of a terrorist bus-bombing attack allowed for graded or staged exposure protocols. Staging in this context can be described as follows: A therapist controls the severity of the scenario and senses stimulated via the pressing of different function keys. Similarly, in a VR delivery requiring user immersion in simulations of trauma-relevant environments, emotional intensity of scenes can be staged, that is, precisely delivered by a clinician personalizing the exposure for the individual patient in a controlled manner (Rizz et al. 2006).

Among others, Josman et al. (2008) and Rizzo et al. (2006) have explored the dynamics, design and the role of multi-sensory staged input in VR applications. Some of the most important research has centered on posttraumatic stress disorder (PTSD) population differential diagnosis, including exploration of syndrome-specific symptomatology, resulting in notable progress. Posttraumatic stress disorder has been defined as follows: "the essential feature of posttraumatic stress disorder (PTSD) is the development of characteristic symptoms following exposure to one or more traumatic events" (DSM-5; American Psychiatric Association 2013, p. 274). Posttraumatic stress disorder in veteran populations is a serious issue, with reports of some 22 veterans taking their lives every day, resulting in a suicide every 65 min (Basu 2013). In August 2012, the US government called for stronger suicide prevention efforts; a year later, the US government announced \$107 million in funding for better mental health treatment for veterans with posttraumatic stress and traumatic brain injury (TBI), notably signature injuries of the wars in Iraq and Afghanistan (Basu 2013).

Church and Brooks (2014) adopted a holistic approach and focused on treatment of spouses of 218 war veterans who were also affected by PTSD; it was reported that following treatment spouses demonstrated substantial symptom reductions. The multi-modal intervention incorporated emotional freedom techniques and other so-called energy psychology methods to address PTSD symptoms. A variety of complementary and alternative medicine modalities for stress reduction and resource building was incorporated (Church and Brooks 2014). Energy psychology has been described as an integrative approach to psychotherapy, coaching and healthcare treatment rooted in mind-body healing traditions that are many thousands of years old. While Church and Brooks (2014) claim some success with this therapeutic approach, it has been argued that energy psychology is a somewhat unsupported and "pseudoscientific" movement (Bakker 2013). It has been argued that there is little empirical support for the theories that inform energy psychology techniques that support for efficacy is methodologically weak and that treatment process has not been able to demonstrate an effect beyond non-specific and/or placebo effects (Bakker 2013).

While therapeutic approach incorporating alternative treatment therapies such as energy psychology is beyond the scope of this review, in terms of future research, it may be nonetheless worthwhile to note these findings and to consider the potential for virtual reality exposure therapy (VRET) treatment of PTSD at an extended familial level. Specifically, this review will focus on olfaction as a variable in virtual reality exposure therapy of PTSD. Notably, a number of studies such as Vasterling et al. (2000) and Dileo et al. (2000) have supported the argument that PTSD patients have significant olfactory deficits. These data provide an opportunity to investigate cognitive aspects of olfactory function in PTSD, leading to the consideration of the use of remedial and therapeutic olfactory stimulation in virtual reality therapy programs.

According to Doty et al. (1997), the first report of posttraumatic anosmia (loss of smell) in the modern literature was Jackson's (1864) description of a 50-year-old man complaining of loss of smell after falling off a horse. Historically, olfactory aspects were noted in the first diagnoses of PTSD in World War I, known at the time as "shell shock." Later, olfaction was a constituent of early VR technology research and development in the 1960s. Despite early presentation, olfaction has apparently been overlooked from a research perspective. This review will consider the connection between emotion, memory and smell. Posttraumatic stress disorder olfactory deficits will be explored, as will delivery of odor stimulation in virtual environments, and a case will be made for olfactory sensory inclusion in VRET. Hypothetically, olfaction as an element of multi-sensory reconstruction in a virtual environment PTSD treatment program may positively impact on the outcome.

\section{Treatment of PTSD}

Therapies to date for PTSD include both traditional and innovative methodologies. Imaginal exposure is a conventional treatment for PTSD, allowing patients to participate in a desensitization process, that is, gradually 
confronting memory of trauma in a supportive therapeutic environment. In 2007, the US Department of Veterans Affairs funded a treatment review of PTSD literature, 2,800 abstracts were identified, 90 randomized clinical trials, 37 pharmacotherapy studies and 53 psychotherapy studies were selected for review. The study confirmed that exposure therapy (ET) was the only treatment considered effective, compared with pharmacotherapy, psychotherapies, cognitive restructuring, coping skills training and group psychotherapy (Institute of Medicine 2007). Exposure therapy has, however, been called a "cruel cure," evoking unpleasant memories and therefore distress in patients (Olatunji et al. 2009); nonetheless, confronting memory of traumatic events is a central tenet of imaginal exposure therapy.

The effectiveness of imaginal exposure therapy has been confirmed (Rothbaum et al. 2000; Rothbaum and Schwartz 2002); however, it has been reported that many military veterans have difficulty retrieving and engaging in traumatic memories long enough to facilitate treatment (Rothbaum et al. 1999). This problem was summarized by Rizzo et al. (2006) as: "avoidance of the reminders of the trauma is inherent in PTSD, and is one of the defining symptoms of the disorder" (p. 236). Additionally, Jaycox, Foa and Morral (1998) identified patient's inability to emotionally engage in imagination process as a predictor for negative PTSD treatment outcomes.

Reger et al. (2011) evaluated the effectiveness of virtual reality exposure therapy (VRET) for 24 active duty soldiers seeking treatment following a deployment to Iraq or Afghanistan. The study showed that virtual reality exposure therapy resulted in significant reductions in PTSD symptoms following an average of seven treatment sessions (Reger et al. 2011). Additionally, 15 (62\%) patients reported clinically meaningful, reliable reduction in PTSD symptoms, thus supporting the effectiveness of exposure therapy for active duty soldiers. These findings were supported by McLay et al. (2012) who tested a method for applying virtual reality exposure therapy to active duty service members diagnosed with combat posttraumatic stress disorder (PTSD). Forty-two service members with PTSD were recruited, 20 participants completed the treatment, it was reported that of those who completed posttreatment assessment, $75 \%$ experienced at least a $50 \%$ reduction in PTSD symptoms. Notably, there were no adverse events associated with VRET treatment, thus providing additional support for the use of VRET in combatrelated PTSD (Reger et al. 2011; McLay et al. 2012).

Virtual reality (VR) treatment of psychological and physical disorders is well established (Glantz et al. 2003; Rizzo et al. 2004). VR has been used to treat traumatized victims from events such as: the Vietnam War (Rothbaum et al. 2001), "September 11" (Difede and Hoffman 2002),
Iraq War (Gerardi et al. 2008) and motor vehicle accident victims (Walshe et al. 2003). VRET has been constructive in facilitating visualization and traumatic memory retrieval (Rothbaum et al. 2001; Vermetten et al. 2007), and use of virtual reality may, therefore, be useful in addressing trauma recall avoidance, thus arguably improving on traditional imagination bound in vivo exposure therapy.

\section{Virtual reality exposure therapy: sense-specific gradual immersion}

Two main virtual reality setups are used to immerse patients in a virtual environment, namely the head-mounted display (HMD) and the computer automatic virtual environment (CAVE). The virtual environment used by Rothbaum et al. (2001), consisted of a basic hovering helicopter simulation, experienced via an HMD, with therapist controlled visual and auditory effects. The Rothbaum et al. (2001) study reported a reduction in PTSD ranging from 15 to $67 \%$; however, there was no control group. Following the attack on the World Trade Centre, Difede and Hoffman (2002) developed a virtual environment with a gradual immersion simulation process. Patients were exposed to explosions, sound effects and virtual subjects jumping from burning towers and reported a significant reduction in PTSD symptoms following treatment; however, it was a small study with only ten participants. Both Rothbaum et al. (2001) and Difede and Hoffman (2002) indicated positive results, albeit with limited participants and some design flaws.

Rizzo et al. (2006) studied the design and development of a virtual Iraq PTSD VR application; notably, olfactory stimuli including the scent of burning rubber, cordite, body odor, diesel fuel, Iraqi spices and gun powder were deployed. While the need to add olfactory and tactile stimuli in VR prototype environments was noted, no data were reported to quantify its effectiveness in terms of gradual staged (step by step) immersion. This gap in the literature was partly addressed by the Josman et al. (2008) study that measured participant distress precipitated by staged sensory VR exposure. Results indicated that the staged addition of sound to visual stimulation elicited emotional responses in subjects incrementally, the more realistic the sensory environment, the greater the emotional response. However, a study of sensory modality in VR therapeutic environments (DiScalfani 2012) reported that overall virtual reality exposure, including visual and auditory stimulation, was sufficient to evoke distress. It was reported that the addition of olfactory and tactile stimulation did not have a significant impact. The authors did, however, note a number of limitations including independent variable considerations and potential experimenter effects (DiScalfani 2012). 
Overall findings support the implementation of sensespecific staged stimuli in VR treatment of PTSD, and the need to make the experience as real as possible. However, the way in which sensory modalities may work together to heighten stimuli sensation in VR PTSD treatment methodologies requires further study.

\section{Olfactory stimulation in virtual reality applications}

The role of visual, auditory and haptic stimulation in VR systems has been established (Rothbaum et al. 2001; Difede and Hoffman 2002; Josman et al. 2008). Olfactory input has had a relatively minor role in VR application and research to date; conversely, olfaction plays a critical role in experiencing the physical world (Chen 2006). Historically, "interest in psychology and olfaction grows annually; traditionally research on vision and audition has tended to dominate behavioural sciences" (Chu and Downes 2000, p. 111). Chen (2006) argues that since inception VR has been overly influenced by visual stimuli, tactile and auditory information have been incorporated; however, olfactory information has been largely ignored as a "minor sensory modality to the virtual environment participant" (Chen 2006, p. 580). This view is supported by Matsukura, Yoneda and Ishida (2013, p. 606) who note that environment is perceived through information that is obtained from sensory systems "most of this information comes from our eyes and ears; therefore, it is natural that most research efforts on virtual reality systems have been devoted to the development of visual and audio displays for the realistic presentation of three-dimensional images and surround sound." Nonetheless, the authors note that the sense of smell is often underestimated when compared with vision, sound and touch, and therefore, may provide some explanatory value as to why comparatively less attention has been paid to the development of olfactory display VR technology (Matsukura et al. 2013).

As outlined, in terms of treatment of PTSD, imaginal exposure therapy is considered the most effective treatment (Institute of Medicine 2007). However, psychological aspects of trauma avoidance mean that recall of the traumatic event is often difficult for patients and may compromise the treatment (Rizzo et al. 2006; Jaycox et al. 1998). Notably, Rizzo et al. (2006) and Josman et al. (2008) reported positive results regarding VR treatment of PTSD. Additionally, sensory stimulation of visual, auditory, haptic and olfactory senses elicited emotional responses in subjects incrementally, the more realistic the sensory environment the greater the emotional response (Rizzo et al. 2006; Josman et al. 2008).

Arguably the key to technological innovation concerning VR treatments of PTSD may lie in the conceptualization of the problem space as a persuasive design issue (Fogg 2009).
Figure 1 represents an adaptation of the Fogg behavior model (Fogg 2009) and considers PTSD population motivation, ability and triggers to recall traumatic events in a diagrammatic context. Factor 1 Motivation to engage in the treatment process: In a military context, research supports that VR solutions are well received by soldiers and preferred over traditional talk therapies (Wilson et al. 2008). Factor 2 Ability: Capability of the soldier to access treatment is a key factor, portable HMD systems make it more likely that treatment will be delivered immediately and in the field (Rothbaum et al. 2001). Factor 3 Triggers: Imaginal exposure therapy centers on the ability of the subject to recall the traumatic event (Rothbaum et al. 1999). VR treatment of PTSD has the potential to allow for staged multi-sensory stimulation via visual, auditory, haptic and olfactory methodologies that can serve as triggers for memory and thus facilitate recall (Larsson 1997). The Fogg (2009) model of persuasive technologies illustrates that higher motivation and ability to participate, coupled with effective triggers, increase the likelihood of generating the target behavior, in this instance recall of the traumatic event for effective treatment purposes.

\section{Virtual reality and PTSD experimental design}

Virtual technology is not a new idea, and Table 1 highlights how VR technology has evolved, building historically on other related technical developments. Sensory stimulation is noted, as are landmark experiments in VR and VRET treatment of PTSD.

Over the past 100 years, many pioneers have helped to develop virtual reality systems, and some highlights are as follows: In the 1890s, Tesla proposed first principles and systems to perform teleoperation; in the 1920s, Link

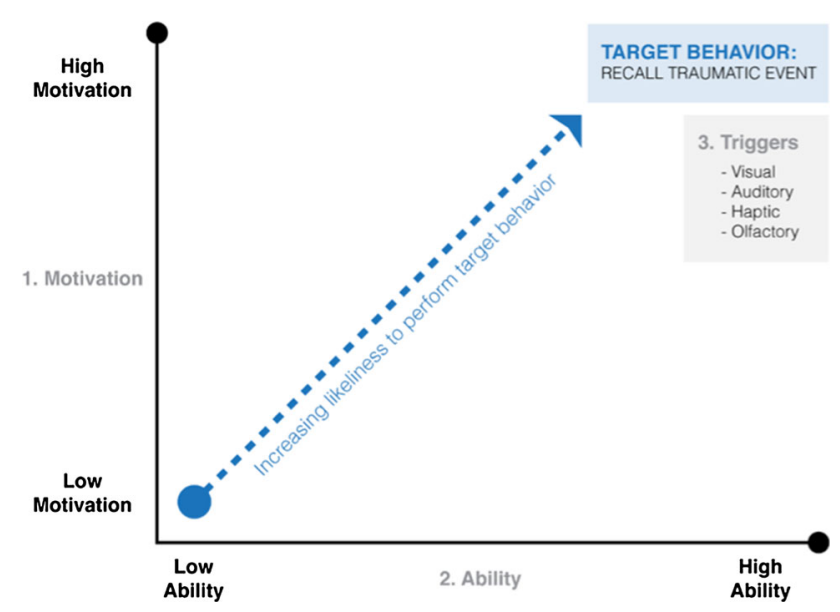

Fig. 1 PTSD VRET target behavior model. Adapted from Fogg (2009) 
Table 1 Overview VR and PTSD experimental design

1890s Nikola Tesla: first principles and systems to perform teleoperation "Method of and Apparatus for Controlling Mechanism of Moving Vessels or Vehicles" (US patent 613,809)

1920s Edwin Link: vehicle simulation-forerunner of virtual reality technology

1940s Teleoperator systems developed to create capabilities for handling highly radioactive material

1950s "Cinerama" developed using three-sided screens. Hans Laube invents the "Smell-O-Vision".

1960s Dr. Ivan Sutherland: synthetic computer-generated displays for virtual environments. Morton Heilig: "Sensorama" immersive, multisensory technology

Philco and Argonne National Laboratory: head-mounted closed circuit TV system, incorporating virtual image viewing optics

1970s The cold war: Numerous military investigations add major contributions to the field of virtual reality, development of flight simulators by NASA

Douglas Engelbart: shapes the future of user interaction via the mouse device

1980s Jaron Lanier: VPL research introduces term virtual reality

NASA: VIVED (Virtual Visual Environmental Display) and VIEW (Virtual Interactive Environment Workstation)

1990s DigiScents: iSmell computer peripheral device, contains cartridge with 128 primary odors—mixed to replicate natural and man-made odors

2000s Rothbaum et al. (2001): VR PTSD study hovering helicopter simulation, experienced via an HMD, therapist controlled visual and auditory effects

Difede and Hoffman (2002): VR PTSD study—gradual immersion simulation process

Haque (2004): "Scent of Space" interactive art installation

ATR Media Laboratories: air cannon type delivers odor in a targeted manner

Thanko (2005): Aroma Generator USB device-three cartridges for different smells

Rizzo et al. (2006): VR PTSD study: "Virtual Iraq" application with olfactory stimuli

Nakaizumi, Yanagida Noma and Hosaka (2006): odor-vapor-trapped vortex rings of air delivered via the air cannon

Yamada (2006): wearable-type display, suitable for HMD, tubes direct odor to the users' nose, compact size

Josman et al. (2008): PTSD VR study measures participant distress precipitated by staged sensory VR exposure

2011 University of California San Diego Jacobs School of Engineering (2011): optimization and miniaturization of component that select and release scents from 10,000 odors, positioned as a digital scent solution for TVs and phones

2013 Matsukura et al. (2013): "Smelling Screen” odor distribution facilitates perception of source

2014 FitzGerald, Richardson and Wesson (2014): implant mice with ipsilateral bipolar electrode in the olfactory tubercle

Facebook (2014): acquisition of virtual reality start-up Oculus VR

Baston (2014): reports experimental design "Birdly” linking Oculus Rift with auditory and olfactory stimulation

developed vehicle simulation, arguably a forerunner of virtual reality technology. The 1950s saw the introduction of "Cinerama" and "Smell-O-Vision." In the 1960s, Ivan Sutherland created the first head-mounted display (HMD) attached to a computer system, and Morton Heilig introduced the Sensorama an early example of immersive, multi-sensory technology (Heilig 1962). In the 1970s, Douglas Engelbart helped to shape the future of user interaction via the mouse device, and Myron Krueger worked with computer graphics and audio in the form of video projection. Jaron Lanier is credited as the person who first coined the term virtual reality in the 1980s. In the 1990s, the "iSmell," a cartridge with 128 primary odors, was developed.

Buxton (1994) maintains that modern technologies have failed to take advantage of all of our human physical abilities, thus perhaps reflecting a distorted view of human senses. For example, standard input devices such as keyboards almost completely fail to take advantage of highly developed human senses such as touch and control over pressure. In terms of olfaction, smells inform regarding immediate vicinity, be that burning toast or fragrant floor polish. Similar to sound, a smell may be strongly tied to a specific source (such as a perfumed flower) or smells may form ambient mixtures in the background (such as the powerful smell of pine trees in a forest). Cater (1992) emphasizes the importance of ambient smell in a physical environment in terms of creating a sense of presence in the virtual environment. Sense of smell is constantly used to inform us about our immediate environment, and therefore logically in a simulated or virtual reality environment, smell plays a key role, and researchers and technology developers should focus on its potential.

In terms of the behavioral sciences, Spooner and Pachana (2006) maintain that replication of everyday life environments in laboratory experiments is crucial as it directly improves validity of results especially concerning subtle interactions. In terms of the focus of this paper, 
replication of real-world stimuli is critical in terms of the research design of VR therapeutic environments. Regarding current VR design, Nakamoto et al. (2008) argue that real-world auditory and visual perceptions are almost perfectly replicated, senses working together to create the overall experience. However, according to Craig et al. (2009), this simulation almost never includes chemosensory perception. Arguably as olfaction is more complex to implement and control (Chen 2006), use in VR environments remains more the exception than the rule. Barfield and Danas (1995) first outlined this fact almost a decade ago, maintaining that olfactory information has been largely ignored as input to virtual environment participants despite the fact that olfactory receptors provide a rich source of information to humans. However, some research to date has incorporated olfactory stimulation, for example, in virtual environments for military training (Vlahos 2006), fire-fighter training and medical diagnosis (Spencer 2006).

Barfield and Danas (1995) define virtual olfactory display as hardware, software and chemicals used to present olfactory information to the virtual environment participant. In order to provide a VR user with a sense of smell, an olfactory display generates a vapor of odorous chemical substances and then delivers it to the user's nose (Matsukura et al. 2013). Scent formulation and delivery are both complex and expensive in virtual environments. Haque (2004) designed "Scent of Space" an interactive art installation incorporating a "Smell System" housed in a gigantic wind tunnel. Smell was delivered to the user by introducing an odor vapor in an airflow field; the large scale of the system arguably limits its application particularly in therapeutic contexts.

Chen (2006) describes ubiquitous-type and wearabletype scent display devices; ubiquitous-type display results in scent delivery to a large area, for example, a CAVE, however, smells linger which makes switching scent difficult, additionally timing of odor delivery and concentration of the delivered odor cannot be precisely controlled (Matsukura et al. 2013). ATR Media Laboratories developed an air cannon-type mechanism to deliver smell in a targeted manner to participants' nostrils, controlled by an interactive application (Yanagida et al. 2004); however, the system was susceptible to breakdown via clogging. Nakaizumi et al. (2006) also designed a system whereby odor was delivered via air cannon. The device emitted odor-vaportrapped vortex rings of air, the collision of two odorcontaining vortex rings proximate to the user's nostrils generating a specific dimensional distribution of the odor. However, Matsukura et al. (2013) point out that odor presentation by this device is discrete in time and that virtual odor sources that continuously release odor vapor cannot be presented with this system.
Wearable-type display, developed by Yamada et al. (2006), is suitable for HMD systems. The system features tubes that direct odor to the users' nose, and the compact size allows the user to walk around in an immersive virtual environment while being presented with odors. The Yamada et al. (2006) system allowed that odor could be switched from one scent to another using computer-controlled solenoid valves, and intensity of odor presented to the user could be altered via through the solenoid valves which had the capacity to dilute odor vapor with clean air.

However, Matsukura et al. (2013) note that a limitation of many olfactory display systems is that most of them simply propel odor vapor directly at the user. They recently proposed a new olfactory display system a Smelling Screen that "could generate odor distribution on a two-dimensional display screen... the generated odor distribution leads the user to perceive the odor as emanating from a specific region of the screen" (Matsukura et al., p. 606). In virtual reality environments, arguably this technical development may help facilitate congruence between the actual VR scene and ambient olfactory odor. Arguably such congruence may be a key factor in the creation of presence in a VR environment, allowing the participant to experience presence (Bystrom et al. 1999; Schubert et al. 2001), that is an actual sensation of "being there" (IJsselsteijn et al. 2000). Presence in VR environments will be further discussed in this paper.

The recent purchase of the virtual reality headset development company Oculus VR, by the social-networking company Facebook, is arguably another significant milestone in the history of VR (Zuckerberg 2014). Multi-sensory stimulation and specifically olfaction would appear to be of interest to social-networking technology companies in a VR experimental context, evidenced by the recent "Birdly" research project which linked Oculus Rift technologies with auditory and olfactory stimulation technologies (Baston 2014). With over one billion users worldwide, significant financial resources and a strong commercial and pioneering ethos, Facebook may perhaps be one of the optimum corporations to help develop VR HMD units that have the capacity to incorporate sophisticated odor display.

Given the complexity of both formulating and delivering scent in VR environments, it may be useful to consider some recent experimental developments in terms of olfactory stimulation. Odors have long been known to deliver degrees of attractiveness or aversion (Locke and Grimm 1949); for example, the odor of burning flesh elicits a repulsive reaction in humans, whereas the smell of freshly baked bread is mostly pleasant (FitzGerald et al. 2014). Control of odor hedonic-driven behaviors requires a fully functional olfactory system, both to detect and discriminate the stimulus, along with the ability to relay this information 
into emotional and reward-related brain structures. The olfactory tubercle (OT) is an olfactory structure with known anatomical connectivity into brain reward structures (Wesson and Wilson 2011). FitzGerald et al. (2014) conducted an interesting experiment consisting of implanting male mice with an ipsilateral bipolar electrode directly into the OT in order to administer electric current and therefore activate this olfactory processing center. The authors of the study confirmed that electrical stimulation of the OT was rewarding, with mice repeatedly self-administering stimulation. Results of this recent experiment perhaps offer some hope in terms of olfactory stimulation in human populations? Perhaps the future of olfactory stimulation of PTSD patients in a VR treatment scenarios may involve bypassing mechanical odor delivery solutions and focusing on direct stimulation of the human olfactory structure utilizing technology to produce the required stimulation.

Evidently, olfactory stimulation mechanisms have been part of the developmental path of various human-centric simulation technologies since the 1950s. Based on the original work of Crocker and Henderson (1927), it has long been maintained that the human olfactory system can detect over 10,000 different smells; however, a recent study published in Science, a journal of original scientific research, has made a remarkable claim that the human nose can actually detect more than one trillion different smells (Bushdid et al. 2014; Davis 2014). The quantitative leap in terms of the order of magnitude of detectable odors from thousands to trillions almost defies intuition. A review of the study methodology reveals that mixtures of 10,20 and 30 components drawn from a collection 128 odorous molecules were employed in the study. Twenty-eight subjects participated and performed forced-choice discrimination tests between pairs of mixtures; each subject completed 264 discrimination tests (260 mixture and four control discrimination tests). The results were reported as follows:

"our results show that humans can discriminate $1.72 \times 1,012$ or $5.58 \times 1,013$ mixtures of 30 components out of the collection of 128 odorous molecules. $1.72 \times 1,012$ may seem like an astonishingly large number. However, there are $1.54 \times 1,029$ possible mixtures of 30 from the 128 components used here. Therefore, if there are $1.72 \times 1,012$ discriminable stimuli, this means that for each mixture tested there will be $8.95 \times 1,016$ other mixtures that cannot be discriminated from it... our results therefore establish only a lower limit of the number of discriminable olfactory stimuli. Although this lower limit of greater than 1 trillion is several orders of magnitude more than distinguishable colors or tones, it is presumably dramatically lower than the actual number of discriminable olfactory stimuli." (Bushdid et al. 2014, p. 1372)
Bushdid et al. (2014) acknowledge that one trillion may seem like "an astonishingly large number," and it is hard to disagree with this sentiment; interestingly, the authors maintain that one trillion may only be a lower limit, and the actual number may be higher. In terms of comparative sensory analysis, for example, in visual and auditory systems, it is estimated that humans can distinguish between 2.3 million and 7.5 million colors (Pointer and Attridge 1998) and 340,000 musical tones (Stevens and Davis 1938). Given the relatively limited number of participants $(\mathrm{N}=28)$ and limited range of odors employed $(\mathrm{N}=128)$, the results of this study are arguably more grounded in complex mathematical probability equations as opposed to large-scale quantitative empirical investigation. Additionally, the authors provide no explanatory detail regarding the significant gap between reported findings to date and the results of their study. Therefore, while interesting, the results should perhaps be interpreted with some caution until follow-up studies have been able to support these findings or not. The difference between the accepted number of discriminable odors cited to date in scientific publications as recently as 2 years ago (Kandel et al. 2013), and new claims of up to one trillion are, however, significant in terms of the marked discrepancy between the figures and therefore points to a need for greater scientific investigation of this phenomenon. Should a trillion or even hundreds of millions of discriminable odors exist, it would certainly present enormous challenges to the designers of VR olfactory display systems.

Stimulus for odor consists of volatile substances mostly lipid soluble and of organic origin, with a molecular weight in the range of 15-300 (Carlson 2010). Sense of smell, known as olfaction, centers on the nose as a sensory organ. In the olfactory system, the olfactory mucous membrane covers a small area in the roof of the nasal cavity and contains olfactory receptor cells which pass scent information to the olfactory cortex in the brain. Diffuse suspensions of molecules, called odors, are analyzed by the nose and are identified by their unique chemical signatures. Human identification and discrimination of odor are complex neurophysiological processes involving aromatic molecules, olfactory receptors, olfactotopic coding and processing of information received from the olfactory bulb to the piriform cortex. In terms of olfaction, Carlson (2010, p. 260) maintains "we do not yet know how maps of chemical structure are combined to form maps of perceptual quality...presumably learning plays some role in the process."

Behavioral scientists often note the paradox of A.I., psychologists arguably do not fully understand the workings of the human brain, so how, therefore, can an artificial intelligence be created if human intelligence is poorly understood? It may in fact be the case that odor simulation 
provides a similar challenge to technologists, progress being dependent on a comprehensive understanding of all mechanisms and phenomena involved. While recent developments by researchers at the University of California San Diego Jacobs School of Engineering (2011) show promise in terms of optimization and miniaturization of technology components releasing scents from 10,000 odors. However, it would appear from the latest research regarding human smell discrimination (Bushdid et al. 2014) that it might require a far greater number of odors in order to realistically replicate real-world olfactory experience.

The Heilig Sensorama delivered an experience of riding a motorcycle in Brooklyn with the wind, vibrations, 3D view, and importantly, smells of the city (Heilig 1962). Paradoxically, olfactory stimulation was very much a part of the early days of immersive technology, yet has to a great extent has been overlooked in contemporary VR research and development (Chen 2006). Creating and delivering scent in a controlled environment has presented both cost and complexity, perhaps delaying extended use of olfactory stimuli in VRET, arguably compounded by the lack of consolidated research to justify time, purpose and investment.

\section{Olfaction and the physiology of behavior}

Clinicians have noted that particular trauma-associated smells, such as napalm or diesel in combat veterans suffering from PTSD, may serve as precipitants of emotional memories and induce traumatic recall (Vermetten et al. 2007). Known as the Proust phenomenon: "Odors are especially powerful reminders of autobiographical experience" (Chu and Downes 2000, p. 111). Second, only to the visual system, a sense heavily utilized in VR, the olfactory cortex receives information from approximately 40 million olfactory receptor cells and is unique in having direct projection to the amygdala. Furthermore, information from olfactory receptors is sent to the hypothalamus, hippocampus and the orbifrontal cortex (Carlson 2010). Known as the limbic system, it is a complex set of structures located on both sides of the thalamus, just under the cerebrum, and includes the hypothalamus, the hippocampus and the amygdala. The limbic system is considered to be responsible for emotional life and specifically the formation of memories.

People often clearly recall a past experience associated with a certain smell "odor-evoked memories...are attributable to the anatomical structure of the brain...the olfactory cortex in the brain has a direct link to the limbic system, which is critical for the experience of emotions and memories" (Matsukura et al. 2013, p. 606). Functional imaging studies indicate that the amygdala in the limbic system plays a role in the formation of emotional memories (Cahil et al. 1996), the amygdala participating in the emotional processing of olfactory stimuli (Mujica-Parodi et al. 2009). Arguably there exists a strong triadic psychophysiological relationship between olfaction, emotion and memory. Smell is capable of altering emotional states (Vermetten et al. 2007) and can facilitate recall (Larsson 1997; Chu and Downes 2000). Additionally, pleasant ambient odors can relieve stress and improve mental relaxation (Lehrner et al. 2000), thereby supporting an argument that stimulating and relaxing odors should be considered in VRET programs.

In terms of olfaction in PTSD and VR treatment, it is perhaps useful to consider victim reports as in this area "odor perception can retrieve memories of life events with personal meaning and elicit strong affective experiences" (Vermetten et al. 2007, p. 9). Murray (2002) describes never being able to forget the smell of burning metal, plastic and people that was in the air for months after the World Trade Towers fell. Winkler (1991) an extract of rape victim testimony reported, "the most gripping body response was smell...the smells convinced me to tell the police of these as part of the rapist's crime" (p. 12). Both accounts cite odor as significant in the traumatic experience, consistent with the intrusive thoughts model that characterize PTSD. These accounts perhaps provide further support of the need to investigate olfaction as a variable in VR treatment of PTSD.

\section{Olfaction and presence in virtual reality exposure therapy}

Virtual environments can affect human experience, producing a sense of physical presence defined as the user's feelings of "being there" in mediated environments (IJsselsteijn et al. 2000). Presence is also defined as the impression of non-mediation, whereby the user no longer perceives the display medium (Lombard and Ditton 1997). Sense of presence is central to psychological research in VE's (Schubert et al. 1999). Interaction is acknowledged as one of the prime facilitators of presence in VE's (Draper et al. 1998; Lombard and Ditton 1997; Steuer 1992); in the context of this paper, olfactory reaction may, therefore, facilitate interaction and thereby increase sense of presence in a VR environment.

However, according to Riva et al. (2007), mediums unable to provoke feelings of presence or immersion may produce low affective response rates. Additionally, Interrante et al. (2012) argue that the relationship between personality, presence and performance in immersive virtual environments (IVE's) is complicated and not easily 
captured by existing measures. However, increasing ambient information can increase perception of presence in VR (Lombard and Ditton 1997). Matsukura et al. (2013) maintain that presenting specific odors to the user of a virtual reality system should create a more realistic experience. Therefore, olfactory stimulation as sensory input may theoretically enhance feelings of presence in VR environments.

The connection between olfaction and emotion has been established (Mujica-Parodi et al. 2009); accordingly, odor can elicit emotions, which may in turn help to facilitate or create a sense of presence. Riva et al. (2007) maintain that: "the experience of presence is a complex multidimensional perception formed through interplay of raw multi-sensory data and various cognitive functions" (p. 46). Multi-sensory implies a role for all senses, vision, audition, touch and logically olfaction. Tangential to the concept of presence lies the notion of self-presence, first introduced by Biocca (1997) to represent "user's model of themselves" inside the virtual world. Biocca (1997) argues that augmenting self-presence, that is, feeling physically or emotionally extended into the virtual environment improves an individual's experience within the environment. Perhaps the introduction of odor variables as employed by Rizzo et al. (2006) particularly customized experience informed odors could enhance the concept of self-presence for PTSD participants in VRET's?

\section{Olfaction identification deficit}

Olfaction can influence emotion and affective response (Vermetten et al. 2007), facilitate recall (Larsson 1997; Chu and Downes 2000), increase sense of presence (Lombard and Ditton 1997) and accordingly may have an important role in VRET. However, two significant studies have raised questions concerning the potential role of olfactory stimulation in VR treatment protocols. Vasterling et al. (2000) conducted a study of Vietnam veterans. Following screening, 68 participants were divided as follows: 26 combat veterans with PTSD diagnosis, 26 combat veterans without mental disorder and 16 non-war zone deployed Vietnam combat veterans without mental disorders. Olfactory identification ability in all subjects was measured using a standardized smell test. Vasterling et al. (2000) found that compared to veterans free of PTSD, veterans diagnosed with PTSD exhibited some olfactory deficit, that is, they exhibited less proficient performance on a wellstandardized olfactory identification test, clinically referred to as microsmia (a lessening ability to smell).

Regarding other conditions, prior research has suggested that olfaction is not affected in mood or other anxiety disorders (Amsterdam et al. 1987). Olfactory processing deficits have, however, been documented in Schizophrenia; for example, Moberg et al. (1997) reported that patients with schizophrenia displayed various dysfunctions specific to different types of olfactory processing. Maternal stress during pregnancy is a known teratogen (a factor that interrupts fetal development) and has been associated with autism spectrum disorders (Science Daily 2001). Bennetto et al.'s (2007) study of 21 participants (aged 10-18 yrs) with autism was compared to 27 matched controls with typical development. Bennetto et al. (2007) found that olfactory identification was significantly worse among participants with autism. Some 1,700 pregnant females were among the many thousand individuals directly exposed to the World Trade Centre attack. Yehuda et al. (2005) established that low cortisol levels were a risk factor for developing PTSD and suggested that traumatic experiences can leave epigenetic marks that may also alter the stress response in offspring (epigenetics: study of heritable changes in gene activity not caused by changes in the DNA sequence). These epigenetic factors could also perhaps explain why some are more susceptible to stress than others and why some people exposed to the World Trade Centre attacks went on to develop PTSD while others did not. Exploration of any potential relationship between olfactory deficits, disparate syndromes and PTSD is beyond the scope of this review; however, the area warrants further investigation; perhaps, trauma is the common denominator?

In terms of Vietnam veterans diagnosed with PTSD, the Vasterling et al. (2000) findings are important; however, the sample size was relatively small $(\mathrm{N}=27)$. Additionally, all veterans had suffered from PTSD for 25-30 years and were older than the non-deployed control group (Vasterling et al. 2000). A number were taking psychotropic medications, hypothesized by some (Schiffman 1983) to affect olfaction; however, this finding does remain inconclusive. Despite some shortcomings, the results are arguably important; the authors of the study did recommend that impulse and anger dyscontrol should also be investigated. A follow-up study of Vietnam veterans diagnosed with PTSD (Dileo et al. 2008) confirmed the Vasterling et al. (2000) findings, corroborating the presence of significant olfaction identification deficits (OIDs) in war veterans with PTSD, compared to a control group. Additionally, OID was identified as a predictor of aggression and impulsivity in veterans suffering PTSD (Dileo et al. 2008). Once again, however, the sample group of veterans with PTSD was small $(\mathrm{N}=31)$. Additionally, findings could not confirm whether OID pre- or postdated the PTSD condition. However, the Sense of Smell Institute (SSI) published a white paper in 2010 addressing the etiology of olfactory dysfunction stating that "in patients with posttraumatic olfactory loss, it is a characteristic that this deficit 
is only noted weeks or even months following the actual incident" (SSI 2010, p. 2). In the case of war veterans with PTSD, the SSI findings would appear to support the construct that OID or related olfactory dysfunction may perhaps postdate the pertinent traumatic event. These findings are important in the consideration of any VRET methodology designed to incorporate some form of olfactory stimulation therapy.

It is necessary to consider the precise relationship between olfactory deficits and PTSD as studies to date have been inconclusive in terms of determining causation; this may be partly explained by the focus on physiological as opposed to psychological aspects of presentation. Vasterling et al. (2000) could not conclude from their findings whether PTSD leads to fronto-limbic dysfunction. Dileo et al. (2008) argued that their study merely contributed to "emerging evidence of orbifrontal dysfunction in the pathophysiology underlying PTSD" (p. 523). In the absence of definitive evidence concerning the causality of olfactory dysfunction related to PTSD, it is necessary to consider further research in this area. In terms of future research, it may be productive to factor in potential psychological contributory factors such as an olfactory-type conversion disorder as discussed in the following section of this paper.

\section{A case for PTSD olfactory conversion disorder?}

Olfactory dysfunction related to PTSD could perhaps be considered in the range of Somatic Symptom and Related Disorders (DSM-5; American Psychiatric Association 2013), as opposed to a discrete mechanical neurotransmitter function affecting the orbifrontal region. Conversion disorders (also known as functional neurological symptom disorders) were common during World War I and II, involving the loss of any sensory modality. Sensory symptoms or deficits are most common in the visual system (blindness), the auditory system (deafness) or insensitivity to feeling (anesthesia). However, there has been little research into the possibility of a form of hysterical microsmia, potentially a major psychological trauma-related conversion disorder of the limbic system. Reviewing the literature, there is little reference to PTSD-induced microsmia as a specific conversion disorder; however, DSM-5; American Psychiatric Association (2013, p. 318) does note the diagnostic criteria of conversion disorder as "one or more symptoms of altered voluntary and sensory motor function...(F44.6) with special sensory symptoms (e.g., visual, auditory, olfactory or hearing disturbance)." There exists an early noteworthy observation by the army psychologist Myers who first used the term "shell shock." Myers' examination of a shell-shocked patient revealed contracted visual fields and a loss of taste and smell, and these symptoms had commenced when shells burst around the soldier (Myers 1915). Notably, olfaction as a distinct variable has been remarked on at early stage of sensory empirical investigation of PTSD.

\section{Olfaction: a staged component of virtual reality exposure therapy}

In order for VR olfaction therapy to be effective, it would arguably be necessary to first of all address the patient's sensory deficits with a remedial VR therapeutic program to attempt to restore a fully functioning sense of smell. Conversion disorders are difficult to treat due to the lack of well-controlled studies; however, Speed (1996) has had some success with positive reinforcement cognitive behavioral therapy (CBT) a structured psychotherapy for depression and modifying dysfunctional thinking and behavior. Arguably a treatment employing similar CBT-type positive reinforcement in a multi-sensory virtual environment could also be considered. A multi-sensory experimental methodology for virtual environment treatment of diagnosed olfactory deficit could be delivered in structured, staged sequential segments. In terms of experimental research design, these segments may perhaps be structured as follows: participants (utilizing head-mounted display system) are guided through a virtual field of pink roses, obtaining haptic feedback by touching the flowers, together with an auditory description of the smell; these stimuli would be accompanied by rose scent display; therefore, visual, haptic, auditory stimulation would be reinforced by olfactory stimulation. The therapeutic VR delivery process could be further enhanced by the use of the Matsukura et al. (2013) "Smelling Screen" display, which would help to localize odor to a specific flower, and thus arguably facilitate identification recall, potentially an important therapeutic step in terms of addressing olfactory identification deficits. It is established that sense of smell influences presence (Chen 2006), such olfactory "sensory localization" may, therefore, facilitate immersion, presence and as such arguably enhance the VR experience of the user (Chen 2006; Riva et al. 2007; Ischer et al. 2014). In terms of VR olfactory display design considerations, Matsukura et al. (2013, p. 607) describe the following:

the grass scent in a meadow is distributed almost uniformly throughout the field since the odor source, i.e., the grass, is uniformly distributed. However, if there is a single rose flower in the meadow, the distribution of its sweet smell is localized around the flower. The intensity of the perceived rose scent changes with one's relative position to the rose, 
which leads to the perception of the odor source location.

The question is as follows: could multi-sensory exposure to a scented entity with localized odor display reawaken neural pathways compromised by trauma or address olfactory identification deficits? In the case of some form of conversion disorder, could an olfactory enhanced VRET provide a dynamic environment for delivery of CBT?

Regarding non-olfaction identification deficit PTSD patients, they could proceed directly to a staged immersion olfaction therapy, delivered in a sensory-rich virtual environment.

Rizzo et al. (2006) proposed highly realistic virtual environments in their treatment design for PTSD. However, Botella et al. (2010) argue that hyperrealism may not be effective, and their findings indicate that customization may produce better results. "Emma's World" an adaptive and flexible VR program designed to treat emotional problems was used by Botella et al. (2010). The VR program allowed therapists to customize unique environments for each participant according to the significance of trauma on an individualized basis. An application such as "Emma's World" could be useful in customizing olfactory input as a staged component of VRET treatment of PTSD. As outlined, VR solutions are well received by soldiers and preferred over traditional talk therapies (Wilson et al. 2008). Accordingly, VR remedial olfactory customized therapy may be useful in rehabilitating and treating patients suffering from PTSD of both military and civilian causation.

Importantly, in terms of psychological trauma, recent reports highlight growing incidents of PTSD in civilian populations who moderate extreme content online for technology companies. This work is increasingly carried out in the Philippines, where moderators are paid as little as a few hundred dollars a month for this work (Chen 2014). This evolving labor force handles content moderation which is the removal of obscene and offensive material for US social-networking sites. Content viewed by moderators includes extreme pornography, violent street fights, animal torture, suicide bombings, child abuse material, horrific traffic accidents and more recently hostage decapitations (Chen 2014) and the burning alive of a pilot captive (Adams 2015). Perhaps now that PTSD has been reported regarding certain work practices in technology industries (Chen 2014), this may now provide some impetus for these companies to invest in technology led therapeutic protocols to address PTSD, which would in turn benefit both civilian and military populations.

Undoubtedly, continuing advances in immersive virtual reality (IVR) technologies (Ischer et al. 2014) open up possibilities in terms of future research. Ischer et al. (2014) maintain that IVR technologies have become a promising framework for immersion, involving more human senses: sight, hearing, touch and notably smell. Ischer et al. (2014) note that as a result of advancement in computer technologies, subject's immersion in three-dimensional (3D) experimental scenarios is improved; therefore, "sense of presence" in the 3D world is increased. "These close-toreality experiences could possess a considerable potential in research, either to obtain better treatment options for people showing behavioral and cognitive deficits or to investigate fundamental hypotheses" (Ischer et al. 2014, p. 2). Sense of smell influences presence (Chen 2006); however, VR environments unable to provoke feelings of presence or immersion may produce low affective psychological response rates (Riva et al. 2007), therefore lack of sense of presence in VR environments may negatively affect therapeutic delivery. Presence and immersion are key constructs within the discipline of cyberpsychology described as the study of the impact of emerging technology on human behavior. According to Yan (2012), this discipline and research approach will enjoy exponential growth in years to come due to continued rapid acceleration of Internet technologies and the unprecedentedly pervasive and profound influence of technology on human beings. Barak and Suler (2008) maintain that scholars from psychology and related fields who join the field of cyberpsychology will undoubtedly contribute to crystallizing new ideas and perhaps to conquering a new scientific frontier.

Arguably a cyberpsychology informed research approach, that is, an inter-disciplinary perspective combining technology and psychology learning's to date may help to inform progress in terms of tackling the problem of VR treatment of PTSD. Additionally, new considerations in terms of cybermethodology may facilitate experimental research design in virtual contexts (Aiken and McMahon 2014).

In terms of technological progress, a lot of emphasis has been placed on replicating real-life auditory and visual perception in VR environments, senses working together to create the overall sensory experience. However, Spence and Gallace (2011, p. 273) note the lack of multi-sensory experience associated with virtual shopping, maintaining that "figuring out how to get "in touch" with the "Websavvy" consumer therefore constitutes one of the most significant challenges for many companies in the marketplace today." Scent display has been developed for use in movie theaters (Nakaizumi et al. 2006), and as a special effect in computer games (Nakamoto et al. 2008). However, of all the senses, olfaction would appear to be one of the most complex to implement and difficult to control (Chu and Downes 2000; Chen 2006), and this is arguably why its use in VR environments remains more the exception than the rule (Ischer et al. 2014). 


\section{Conclusions}

Evaluation of the role of sensory stimulation in the field of VR has highlighted olfactory stimulation as a potentially powerful yet underutilized therapeutic protocol. Historically, olfactory deficit has been noted as a component of PTSD (Myers 1915), and notably, early designs incorporated smell in the virtual experience (Mortonheilig.com 2010). However, arguably olfaction in virtual environment design and development has failed to maintain a position commensurate with its sensory capacity (Chu and Downes 2000; Chen 2006; Ischer et al. 2014), exemplified by the paucity of research, application and likely associated development cost factors.

Researchers have explored a multiplicity of VR applications suitable for a wide range of the traumatic event victims (Rothbaum et al. 2001; Difede and Hoffman 2002; Walshe et al. 2003; Gerardi et al. 2008; Josman et al. 2008); however, only one major study (Rizzo et al. 2006) incorporated odor as a variable, nonetheless failing to collect data relating to its efficacy. Two studies have, however, highlighted a powerful relationship between olfactory dysfunction and PTSD (Vasterling et al. 2000; Dileo et al. 2008). Results indicate that a significant cohort of the PTSD population suffer from olfactory dysfunction that clearly must be addressed before any stimulation, or exposure therapy can be considered. These studies have presented a major consideration in the treatment of olfaction identification deficit PTSD patients. Nevertheless, findings provide scope for the exploration of remedial olfactory therapeutic programs (Lehrner et al. 2000), incorporating staged (Josman et al. 2008), customized (Botella et al. 2010), and localized (Matsukura et al. 2013) olfactory stimulation and additionally facilitating recall (Larsson 1997; Chu and Downes 2000; Rothbaum et al. 2001; Vermetten et al. 2007), evoking emotion (Vermetten et al. 2007; Mujica-Parodi et al. 2009), creating presence (Lombard and Ditton 1997; Biocca 1997), and facilitating immersion (Difede and Hoffman 2002; Matsukura et al. 2013; Ischer et al. 2014). The literature supports that such an environment is likely to result in positive treatment outcomes (Riva et al. 2007; Ischer et al. 2014).

Findings suggest that olfaction as a component of VRET may be productive in terms of treatment of PTSD; however, research must be undertaken in the formulation, display, staging, customization and localization of scent, coupled with an in-depth analysis of the role of olfaction in cognitive function, emotion, creation of memory and recall. Importantly, Majid and Burenhult (2014) have challenged the notion that humans cannot verbalize their experience of various smells. In their study of the Jahai people of Malay, they found Jahais could express smells in words at a much higher level of accuracy than their
English-speaking counterparts, arguing that the assumption that people are bad at naming smells is not universally valid. "Odors are expressible in language, as long as you speak the right language" (Majid and Burenhult 2014, p. 266); however, whether any language has the word power to describe millions or a trillion odors is a debatable question.

Furthermore, research is required into causative or reactive mechanisms that may underlie olfactory deficit in PTSD and perhaps other disparate syndromes that present olfactory dysfunction. Undoubtedly, there will be continued debate as to the effectiveness of olfaction in virtual reality. Arguably the literature supports a hypothesis that olfaction as an element of multi-sensory reconstruction in a virtual environment PTSD program may have a positive impact. That is, with a proviso that research be undertaken to maximize the potential and effectiveness of olfaction as a variable in any form of virtual reality exposure therapy.

The authors have reviewed available technologies in terms of odor delivery systems; there are many challenges when considering the type of scents that may be required in the treatment of PTSD. Typically, different odors are created from a limited number of scents (Matsukura et al. 2013); however, VR delivery of unique battlefield simulation ranging from burning flesh to the chemical smells of explosive devices (Rizzo et al. 2006) undoubtedly present specific challenges. Head-mounted display units offer promising results in terms of VR PTSD treatment in situ (Rothbaum et al. 2001), portability being a key requirement in conflict scenarios. Recent developments such as the "Smelling Screen" (Matsukura et al. 2013) may offer greater opportunity in terms of enhancing immersion in any VR PTSD treatment protocol; however, the size of the current apparatus may limit its application in the field. Perhaps a microversion of the "Smelling Screen" technology incorporated into an HMD unit may offer the best combination of both methodologies? The entry of the behemoth Facebook into the VR HMD market may offer significant opportunities for research, investment and technological developments in this area. Employment of the Fogg (2009) model in the development of persuasive technologies may also help to illuminate the research process; however, further research will be required with PTSD population to test this premise. Additionally, it may be interesting to bypass the highly complex process of scent formulation, delivery and display and explore direct electrical stimulation of the olfactory tubercle (FitzGerald et al. 2014). Perhaps some form of electrical stimulation technology could be designed within a VR HMD unit and therefore could automatically deliver olfactory stimulation and simultaneously address some of the existing and ongoing limitations of mechanical odor formation and delivery systems. 
As always there are cost implications in terms of researching and developing new technical prototypes, future research projects are complex given the vulnerable nature of the specific PTSD population under study. Emergence of apparent online content moderation-induced PTSD arguably provides impetus for technology companies to engage actively in researching and developing VR therapeutic protocols. Given likely cost-efficiencies of technology facilitated early intervention immersive virtual reality multi-sensory therapy, versus long-term standard treatment of PTSD, further innovative research approaches are undoubtedly commercially and scientifically worthy of exploration. Following their \$2bn acquisition by Facebook, the founders of Oculus VR have predicted that in the next decade, virtual reality will become ubiquitous, affordable and transformative (Dredge 2014). Hopefully, the adoption of a human-centric technological research approach can be equally transformative and will positively impact on the delivery of VR exposure therapy to PTSD population, a Lickliderian symbiosis of man and machine in scientific investigation.

Conflict of interest The authors declare that there is no conflict of interest.

Open Access This article is distributed under the terms of the Creative Commons Attribution License which permits any use, distribution, and reproduction in any medium, provided the original author(s) and the source are credited.

\section{References}

Adams P (2015) Jordan pilot hostage Moaz al-Kasasbeh 'burned alive'. Retrieved 6 February 2015. http://www.bbc.com/news/ world-middle-east-31121160

Aiken MP, Mc Mahon C (2014) A primer on research in mediated environments: reflections on cybermethodology. Report. Dublin, Ireland. Retrieved Jan 4th from https://cypsy.com/a-primer-onresearch-in-mediated-environments-reflections-on-cybermethodol ogy/cybermethodology/

Ischer MJ et al (2014) How incorporation of scents could enhance immersive virtual experiences. Front Psychol 5. http://www. frontiersin.org/Journal/Abstract.aspx?s=196\&name=cognitive_ science\&ART_DOI=10.3389/fpsyg.2014.00736

American Psychiatric Association (2013) Diagnostic and statistical manual of mental disorders, 5th edn. American Psychiatric Publishing, Arlington

Amsterdam JD, Settle G, Doty RL, Abelman E, Winokur A (1987) Taste and smell perception in depression. Biol Psychiatry 22:1477-1481

Bakker GM (2013) The current status of energy psychology: extraordinary claims with less than ordinary evidence. Clin Psychol 17(3):91-99. doi:10.1111/cp.12020

Barak A, Suler J (2008) Psychological aspects of cyberspace: theory, research, applications. Cambridge University Press, Cambridge

Barfield W, Danas E (1995) Comments on the use of olfactory displays for virtual environments. Presence 5(1):109-121

Basu M (2013) Why suicide rate among veterans may be more than 22 a day. CNN News. Retrieved 20th January 2014 from http:// edition.cnn.com/2013/09/21/us/22-veteran-suicides-a-day/
Baston J (2014) The oculus rift made me believe i could fly. Retrieved 6 February 2015. http://www.wired.com/2014/08/oculus-riftbirdly-fly/

Bennetto L, Kuschner ES, Hyman SL (2007) Olfaction and taste processing in autism. Biol Psychiatry 62:1015-1021. doi:10. 1016/j.biopsych.2007.04.019

Biocca F (1997) The cyborg's dilemma: progressive embodiment in virtual environments. J Comput-Med Commun. 3(2). http://online library.wiley.com/doi/10.1111/j.1083-6101.1997.tb00070.x/full

Botella C, García-Palacios A, Guillen V, Baños RM, Quero S, Alcaniz M (2010) An adaptive display for the treatment of diverse trauma PTSD victims. Cyberpsychol Behav Soc Netw 13(1):67-71

Bushdid C, Magnasco MO, Vosshall LB, Keller A (2014) Humans can discriminate more than 1 trillion olfactory stimuli. Science 343(6177):1370-1372. Available at: http://www.sciencemag. org/content/343/6177/1370.abstract

Buxton W (1994) The three mirrors of interaction: a holistic approach to user interfaces. In: MacDonald LW, Vince J (eds) Interacting with virtual environments. Wiley, New York

Bystrom K, Barfield W, Hendrix C (1999) A conceptual model of the sense of presence in virtual environments. Presence. 8(2):241-244 (serial online). Academic Search Complete, Ipswich, MA. Accessed 4 Jan 2015

Cahil L, Haier RJ, Fallon J, Alkire MT et al (1996) Amygdala activity at encoding correlated with long term free recall of emotional memory. Proc Natl Acad Sci USA 93:8016-8021

Carlson NR (2010) Physiology of behaviour, 10th edn. Pearson, Boston

Cater JP (1992) The nose have it! Letters to the editor. Presence 1(4):493-494 (Fall 1992)

Chen Y (2006) Olfactory display: development and application in virtual reality therapy. In: Proceedings of the 16th international conference on artificial reality and telexistence, Hangzhou, China, pp 580-584

Chen A (2014) Content moderation. Retrieved December 20th 2014 from http://www.wired.com/2014/10/content-moderation/

Chu S, Downes J (2000) Odour-evoked autobiographical memories: psychological investigations of Proustian phenomena. Chem Senses 25:111-116

Church D, Brooks AJ (2014) CAM and energy psychology techniques remediate PTSD symptoms in veterans and spouses. Explore $10(1): 24-33$

Craig AB, Sherman WR, Will JD (2009) Developing virtual reality applications: foundations of effective design. Morgan Kaufmann; Elsevier Science distributor, Burlington, MA; Oxford

Crocker EC, Henderson LF (1927) Analysis and classification of odors: an effort to develop a workable method. Am Perfum Essent Oil Rev 22:325

Davis N (2014) Human nose can detect more than 1 trillion smells, scientists discover. Retrieved 20th December from http://www. theguardian.com/science/2014/mar/20/human-nose-detect-1-tril lion-smells-odours

Difede J, Hoffman H (2002) Virtual reality exposure therapy for World Trade Center posttraumatic stress disorder: a case report. CyberPsychology \& Behavior 5:529-535

Dileo FJ, Brewer M, Hopwood V, Anderson V, Kramer M (2008) Olfactory identification dysfunction, aggression and impulsivity in war veterans with post-traumatic stress disorder. Psychol Med 38:523-531. doi:10.1017/S0033291707001456

Discalfani JM (2012) Enhancing virtual reality exposure with olfactory and tactile cues. Ph.D. Dissertation, Hofstra University. Advisor(s) Mitchell LS. AAI3549428

Doty RL, Yousem DM, Pham LT, Kreshak AA, Geckle R, Lee WW (1997) Olfactory dysfunction in patients with head trauma. J Arch Neurol 54:1131-1140 
Draper JV, Kaber DB, Usher JM (1998) Telepresence. Hum Factors 40:354-375

Dredge S (2014) Facebook closes its \$2bn Oculus Rift acquisition. What next? Retrieved November 30th from http://www.theguar dian.com/technology/2014/jul/22/facebook-oculus-rift-acquisitionvirtual-reality

FitzGerald BJ, Richardson K, Wesson DW (2014) Olfactory tubercle stimulation alters odor preference behavior and recruits forebrain reward and motivational centers. Front Behav Neurosci 8. http:// www.frontiersin.org/Journal/Abstract.aspx?s=99\&name=behavioral_ neuroscience\&ART_DOI=10.3389/fnbeh.2014.00081

Fogg BJ (2009). A behavior model for persuasive design. In: Proceedings of the 4th international conference on persuasive technology (Persuasive '09). ACM, New York, NY, USA, Article 40, 7 pp. doi:10.1145/1541948.1541999

Gerardi M, Rothbaum BO, Ressler K et al (2008) Virtual reality exposure therapy using a virtual Iraq: case report. J Trauma Stress 21:209-213

Glantz K, Rizzo AA, Graap K (2003) Virtual reality for psychotherapy: current reality and future possibilities. Psychotherapy 40(1/2):55-67

Haque U (2004) Scent of space: an interactive smell system. In: Proceedings of SIGGRAPH, p 35. doi:10.1145/1186223.1186267

Heilig ML (1962) Sensorama simulator. U.S. Patent 3050870 , August 28, 1962

IJsselsteijn W, deRidder H, Freeman J, Avons SE (2000). Presence: concept, determinants and measurement. In: Proceedings of the SPIE, human vision and electronic imaging V (pp. 3959-3976). Presented at photonics west-human vision and electronic Imaging V, San Jose, USA, January 23-28

Interrante V, Kaeding M, Ries B, Anderson L (2012) Correlations between physiological response, gait, personality, and presence in immersive virtual environments. Presence 21(2):119-141. doi:10.1162/PRES_a_00100

Jackson JH (1864) Illustrations of diseases of the nervous system. Lond Hosp Rep 1:470-471

Jaycox LH, Foa EB, Morral AR (1998) Influence of emotional management habituation on exposure therapy for PTSD. J Consult Clin Psychol 66:186-192

Josman N, Reisberg A, Weiss PL, Garcia-Palacios A, Hoffman HG (2008) CyberPsychol Behav 11(6):775-777. doi:10.1089/cpb. 2008.0048

Kandel ER, Schwartz JH, Jessell TM, Siegelbaum SA, Hudspeth AJ (2013) Principles of neural science, 5th edn. McGraw-Hill Companies, New York

Larsson M (1997) Semantic factors in episodic recognition of common odors in early and late adulthood: a review. Chem Senses 22:623-633

Lehrner J, Eckersberger C, Walla P (2000) Ambient odor of orange in a dental office reduces anxiety and improves mood in female patients. Physiol Behav 10:1-15

Locke B, Grimm CH (1949) Odor selection, preferences and identification. J Appl Psychol 33:167-174. doi:10.1037/h0062514

Lombard M, Ditton T (1997) At the heart of it all: the concept of presence. J Comput Med Commun 3(2). doi:10.1111/j.10836101.1997.tb00072.x

Majid A, Burenhult N (2014) Odors are expressible in language, as long as you speak the right language. Cognition 130(2):266-270. doi:10.1016/j.cognition.2013.11.004

Matsukura H, Yoneda T, Ishida H (2013) Smelling screen: development and evaluation of an olfactory display system for presenting a virtual odor source. IEEE Trans Vis Comput Graph 19(4):606-615

McLay RN, Graap K, Spira J, Perlman K, Johnston S, Rothbaum BO, Difede JA, Deal W, Oliver D, Baird A, Bordnick PS, Spitalnick J, Pyne JM, Rizzo A (2012) Development and testing of virtual reality exposure therapy for post-traumatic stress disorder in active duty service members who served in Iraq and Afghanistan. Mil Med 177(6):635-642

Moberg PJ, Doty RL, Turetsky BI, Arnold SE, Mahr RN, Gur RC, Bilker W, Gur RE (1997) Olfactory identification deficits in schizophrenia: correlation with duration of illness. Am J Psychiatry 154:1016-1018

Mortonheilig.com (2010) Inventor in the field of Virtual Reality. Retrieved October 10th from: http://www.mortonheilig.com/ InventorVR.html

Mujica-Parodi L, Strey H, Frederick B, Savoy R, Cox D et al (2009) Chemosensory cues to conspecific emotional stress activate amygdala in humans. PLoS ONE 4(7):e6415. doi:10.1371/ journal.Pone.0006415

Murray G (2002) I will never forget the smell. The Globe and Mail. November 12, 2010 Retrieved September 10th from http://www. ctv.ca/special/sept11/hubs/newyork/murray

Myers CS (1915) A contribution to the study of shellshock. Being an account of the cases of loss of memory, vision, smell and taste admitted to the Duchess of Westminster's War Hospital. Le Touquet. Lancet 185:316-320

Nakaizumi F, Yanagida Y, Noma H, Hosaka K (2006) Spotscents: a novel method of natural scent delivery using multiple scent projectors. In: Proceedings on IEEE Virtual Reality, pp 213-218. doi:10.1109/VR.2006.122

Nakamoto T, Otaguro S, Kinoshita M, Nagahama M, Ohinishi K, Ishida $\mathrm{T}$ (2008) Cooking up an interactive olfactory game display. IEEE Comput Graph Appl 28(1):75-78. doi:10.1109/ MCG.2008.3

National Academies of Science Institute of Medicine Committee on Treatment of Posttraumatic Stress Disorder (2007) Treatment of posttraumatic stress disorder: an assessment of the evidence. ISBN:0-309-10925-6. Retrieved from http://www.iom.edu

Olatunji BO, Deacon BJ, Abramowitz JS (2009) The cruelest cure? Ethical issues in the implementation of exposure-based treatments. Cogn Behav Pract 16:172-180

Pointer MR, Attridge GG (1998) The number of discernible colours. Color Res Appl 23:52-54

Reger GM, Holloway KM, Candy C, Rothbaum B, Difede J, Rizzo AA, Gahm GA (2011) Effectiveness of virtual reality exposure therapy for active duty soldiers in a military mental health clinic. J Trauma Stress 24(1):93-96

Riva G, Mantovani F, Capideville CS, Preziosa A, Morganti F, Villani D, Gaggioli A, Botella C, Alcaniz M (2007) Affective interactions using virtual reality: the link between presence and emotions. Cyberpsychol Behav 10(1):45-56

Rizzo AA, Schultheis MT, Kerns K, Mateer C (2004) Analysis of assets for virtual reality applications in neuropsychology. Neuropsychol Rehabil 14(1):207-239

Rizzo A, Pair J, Graap K, Manson B, McNerney P, Wiederhold M et al (2006) A virtual reality exposure therapy application for Iraq war military personnel with post traumatic stress disorder. In: Roy M (ed) Novel approaches to the diagnosis and treatment of posttraumatic stress disorder. IOS Press, Amsterdam, pp 235-250

Rothbaum BO, Schwartz AC (2002) Exposure therapy for posttraumatic stress disorder. Am J Psychother 56:59-75

Rothbaum BO, Hodges L, Alarcon R et al (1999) Virtual reality exposure therapy for PTSD Vietnam veterans: a case study. J Trauma Stress 12:263-271

Rothbaum BO, Meadows EA, Resick P et al (2000) Cognitivebehavioral therapy. In: Foa EB, Keane TM, Friedman MJ (eds) Effective treatments for PTSD. Guilford, New York, pp 60-83

Rothbaum BO, Hodges L, Ready D et al (2001) Virtual reality exposure therapy for Vietnam veterans with posttraumatic stress disorder. J Clin Psychiatry 62:617-622 
Schiffman SS (1983) Taste and smell in disease olfactory identification of PTSD. NewEngl J Med 308:1275-1278

Schubert TW, Friedmann F, Regenbrecht H (2001) The experience of presence: factor analytic insights. Presence 10:266-281

Science Daily (2001) Major stress during pregnancy. Retrieved December 30, 2013 from http://www.sciencedaily.com/releases/ 2001/11/011127005411.htm

Sense of Smell Institute (2010) Quality of life in olfactory dysfunction Retrieved December 30, 2013, from http://www.senseofs mell.org/feature/whitepaper/whitepaper_print.html

Spence C, Gallace A (2011) Multisensory design: reaching out to touch. Psychol Market 28(3):267-308

Spencer BS (2006) Incorporating the sense of smell into patient and haptic surgical simulators. IEEE Trans Inf Technol Biomed 10(1):168-173

Spooner DM, Pachana NA (2006) Ecological validity in neuropsychological assessment: a case for greater consideration in research with neurologically intact populations. Arch Clin Neuropsychol 21:327-337. doi:10.1016/j.acn.2006.04.004

Steuer J (1992) Defining virtual reality: dimensions determining telepresence. J Commun 42:72-92

Stevens SS, Davis HH (1938) Hearing, its psychology and physiology. Wiley, New York, pp 152-154

University of California San Diego Jacobs School of Engineering. (2011). Coming to TV screens of the future: a sense of smell. Retrieved from: http://www.jacobsschool.ucsd.edu/news/news_ releases/release.sfe?id=1082

Vasterling JJ, Brailey K, Sutker PB (2000) Olfactory identification in combat-related posttraumatic stress disorder. J Trauma Stress 13:241-253. doi:10.1023/A:1007754611030

Vermetten E, Schmahl C, Southwick SM, Bremner JD (2007) Positron tomographic emission study of olfactory induced emotional recall in veterans with and without combat-related posttraumatic stress disorder. Psychopharmacol Bull 40(1):8-30 Vlahos J (2006) The smell of war. Popular Sci 8

Walshe DG, Lewis EJ, Kim SI et al (2003) Exploring the use of computer games and virtual reality in exposure therapy for fear of driving following a motor vehicle accident. Cyber-Psychol Behav 6:329-334

Wesson DW, Wilson DA (2011) Sniffing out the contributions of the olfactory tubercle to the sense of smell: hedonics, sensory integration and more? Neurosci Biobehav Rev 35:655-668. doi:10.1016/j.neubiorev.2010.08.004

Wilson JAB, Onorati K, Mishkind M, Reger MA, Gahm GA (2008) Soldier attitudes about technology-based approaches to mental healthcare. Cyberpsychol Behav 11(6):767-769

Winkler C (1991) Rape as social murder. Anthropol Today 7(3):12-14

Yamada T, Yokoyama S, Tanikawa T, Hirota K, Hirose M (2006) Wearable olfactory display: using odor in outdoor environment. IEEE virtual reality 2006, pp 199-206

Yan Z (2012) Encyclopedia of cyber behaviour. IGI Global. http:// www.amazon.com/Encyclopedia-Cyber-Behaviour-Zheng-Yan/ dp/1466603151. Aaccessed 4 May 2013

Yanagida Y, Kawato S, Noma H, Tomono A, Tetsutani N (2004) Projection-based olfactory display with nose tracking. In: Proceedings from IEEE virtual reality, pp 43-50

Yehuda R, Engel SM, Brand SR, Seckl, J., Marcus SM, Berkowitz GS (2005) Transgenerational effects of posttraumatic stress disorder in babies of mothers exposed to the World Trade Center attacks during pregnancy. J Clin Endocrinol Metab 90(7):4115-4118. http://www.ncbi.nlm.nih.gov/pubmed/15870120. Accessed 17 Jan 2014

Zuckerberg M (2014) Retrieved from https://www.facebook.com/ zuck/posts/10101319050523971 